\title{
Pharmacometric approach to assist dosage regimen design in neonates undergoing therapeutic hypothermia
}

\author{
Saikumar Matcha ${ }^{1}$, Elstin Anbu Raj ${ }^{1}$, Ramya Mahadevan ${ }^{2}$, Arun Prasath Raju ${ }^{1}$, V Rajesh ${ }^{1}$, Leslie Edward Lewis ${ }^{3}$ and \\ Surulivelrajan Mallayasamy ${ }^{1 凶}$
}

(c) The Author(s) 2021

BACKGROUND: Therapeutic hypothermia (TH) is the treatment of choice for neonates diagnosed with perinatal asphyxia (PA). Dosing recommendations of various therapeutic agents including antimicrobials were not specifically available for the neonates undergoing $\mathrm{TH}$.

METHODS: A systematic search methodology was used to identify pharmacokinetic (PK) studies of antimicrobials during TH. Antimicrobials with multiple PK studies were identified to create a generalizable PK model. Pharmacometric simulations were performed using the PUMAS software platform to reproduce the results of published studies. A suitable model that could reproduce the results of all other published studies was identified. With the help of a generalizable model, an optimal dosage regimen was designed considering the important covariates of the identified model.

RESULTS: With the systematic search, only gentamicin had multiple PK reports during TH. A generalizable model was identified and the model predictions could match the reported/observed concentrations of publications. Birth weight and serum creatinine were the significant covariates influencing the PK of gentamicin in neonates. A dosage nomogram was designed using pharmacometric simulations to maintain gentamicin concentrations below $10 \mu \mathrm{g} / \mathrm{mL}$ at peak and below $2 \mu \mathrm{g} / \mathrm{mL}$ at trough.

CONCLUSIONS: A generalizable PK model for gentamicin during TH in neonates was identified. Using the model, a dosing nomogram for gentamicin was designed.

Pediatric Research (2022) 92:249-254; https://doi.org/10.1038/s41390-021-01714-0

\section{IMPACT:}

- Dosing guidelines for antimicrobials during TH in neonates is lacking.

- This is the first study to identify the generalizable model for gentamicin during TH in neonates.

- Nomogram, proposed in the study, will aid the clinicians to individualize gentamicin dosing regimen for neonates considering the birth weight and serum creatinine.

\section{INTRODUCTION}

Perinatal asphyxia (PA) is a condition in which the newborn is deprived of oxygen during the birth process. ${ }^{1}$ PA affects four million newborns each year, resulting in one million neonatal deaths worldwide. ${ }^{1}$ Neonates with PA are more likely to develop encephalopathy and acute renal injury, as well as other infections, because of their extended stay in the hospital. ${ }^{2,3}$ For newborns with PA, therapeutic hypothermia $(\mathrm{TH})$ is the recommended treatment. $\mathrm{TH}$ is a $72-\mathrm{h}$ process in which the core body temperature is maintained at $34-33^{\circ} \mathrm{C}$, followed by a $6-\mathrm{h}$ rewarming phase to normal body temperature. ${ }^{4-6} \mathrm{TH}$ can lead to a number of physiological abnormalities affecting various organs including kidneys, leading to the accumulation of toxins and drugs. ${ }^{7-9}$ PA is managed with $\mathrm{TH}$, along with antiepileptics, antimicrobials, and other medications, depending on the complications. $^{10}$
Antimicrobial prescribing guidelines for newborns are available; however, its use during $\mathrm{TH}$ is still a challenge. One of the primary reasons for the complexity of dosing guidelines appears to be the lack of pharmacokinetic (PK) data during TH. To prevent subtherapeutic or toxic effects, antimicrobials should be maintained within the therapeutic window.

Population PKs (PopPKs) is a pharmacometric discipline that facilitates in identifying and accounting for sources of variability. ${ }^{11}$ A pharmacometric approach can be used to simulate the time vs. concentration profile of a drug for an individual, taking into account the significant covariates that were found to influence the PK parameters. ${ }^{12}$ Antimicrobial dosing regimens for asphyxiated infants undergoing $\mathrm{TH}$ are not available. Due to the reduced clearance $(\mathrm{CL})$ of several antimicrobials during $\mathrm{TH}$, the standard dosing regimen used in neonates may result in toxic concentrations. The aim of this study was to conduct a thorough analysis of

\footnotetext{
${ }^{1}$ Department of Pharmacy Practice, Manipal College of Pharmaceutical Sciences, Manipal Academy of Higher Education, Manipal, Udupi, Karnataka, India. ${ }^{2}$ PUMAS-Al, Inc., Baltimore, MD, USA. ${ }^{3}$ Department of Paediatrics, Kasturba Medical College, Manipal Academy of Higher Education, Manipal, Udupi, Karnataka, India. ${ }^{\circledR}$ email: msv.rajan@manipal. edu
} 
the PK of various antimicrobials prescribed during TH in newborns diagnosed with PA and to use meta-model approach and pharmacometric simulations to identify generalizable models. The dosage recommendation for this population will be provided based on the identified generalizable model

\section{METHODS}

We used previously reported PopPK models in neonates to generate virtual participants for use in the simulations in this investigation. No institutional review board approval was required for this study as no patient data were used. The species used in the study are the virtual neonatal population.

\section{Systematic review}

Electronic databases such as Scopus, PubMed, Medline, and CINAHL were used to conduct a systematic search. The following keywords: "infant*" OR "neonate" ${ }^{*}$ OR "newborn" ", "asphyxia neonatorum" ${ }^{*}$ OR "birth asphyxia*" OR "Perinatal asphyxia" OR "hypoxic ischemic encephalopathy", "therapeutic hypothermia" OR "induced hypothermia" OR "controlled hypothermia" OR "cooling therapy", "Anti-Bacterial agent*" OR "Anti-Infective Agent" has been used to build the search strategy across the databases The studies that were included were completed before June 2020. The entire search approach is described in Table 1. PRISMA rules were followed when conducting the search.

The screening was performed simultaneously by two reviewers (S.M. and E.A.R.) based on the inclusion criteria. To include the relevant publications, the abstracts were screened first, followed by full-text screening. For any further inclusion, the references of the included articles were also reviewed. Any disagreements that arose during the process were resolved through discussion. Two reviewers worked on data extraction concurrently and independently, using a predesigned data form to capture appropriate data pertinent to the review. The final study included PK and PopPK, which monitored primary PK parameters such as $\mathrm{CL}$, volume of distribution $\left(V_{\mathrm{d}}\right)$, and additional parameters such as elimination rate constant $\left(K_{\mathrm{e}}\right)$, and halflife $\left(T_{1 / 2}\right)$. In the instance where two reviewers disagreed, a third reviewer (S.R.M.) was contacted to provide clarification. The evaluation includes prospective and retrospective, interventional and observational studies that investigated the PK of antimicrobial agents in neonates during $\mathrm{TH}$. Nonhuman studies and non-English language studies were excluded.

\section{Pharmacometric simulations for identifying a generalizable model}

A minimum of two PK studies were required for any drug to be used in the derivation of a generalizable model. The analysis includes PK studies of antimicrobial drugs that met the inclusion criteria. The investigations collected all available demographics and data, including peak concentration $\left(C_{\text {max }}\right)$, trough concentration $\left(C_{\min }\right)$, and other PK parameters. PopPK studies were discovered among the studies included in the systematic review. Using the mean and median demographic data from the individual research, a typical subject representing that study's population was created. For that population, the reported average dose was used as the normal dose. The characteristics of the PopPK models from selected studies were used to generate antimicrobial agent PK profiles. The replicated model was validated by simulating peak and trough concentrations from each study.

A PopPK model was chosen as a reference model, and simulations were performed using demographic data from the other studies to compare peak and trough values reported in the other studies. This process was repeated until all of the reported studies were compared to the published models. As a generalizable model, a definite model was chosen that could match the reported values of all or the majority of the studies.

Following the selection of a generalized model, pharmacometric simulations with relevant covariates were performed. All of the studies provided a set of covariate values that were tabulated. Using pharmacometric simulations, the optimal dosage regimen for individuals representing these tabulated covariate values was screened. Based on these simulations, an optimal dosage regimen for all individuals within the range of covariates was provided in the table. The Julia computing language's PUMAS package version 1.1.0 was used to perform all of the simulations. ${ }^{13}$

\section{RESULTS}

A total of ten studies met the inclusion criteria. The use of gentamicin, amikacin, amoxicillin, and ampicillin for newborns during $\mathrm{TH}$ has been studied. Demographic details such as gestational age (GA), birth weight (BWT), and PK parameters like $\mathrm{CL}$ and $V_{\mathrm{d}}$ during $\mathrm{TH}$ from various studies are presented in Table 1. The PopPK approach was utilized to estimate PK parameters for the studies, which reported a PopPK model. PopPK modeling was performed in these studies using software packages such as NONMEM and nonparametric pharmacometric modeling and simulation package in R. Effect of various covariates on parameters and interindividual variability/between-subject variability of parameters was also reported in these studies. In these studies, the model was qualified using the bootstrap resampling process and visual prediction checks. The impact of $\mathrm{TH}$ on $\mathrm{CL}, V_{\mathrm{d}}$ and the outcomes of the study are shown in Table 1. Since there were seven PK trials to compare, gentamicin models were taken further to identify a generalizable model. Other drugs were left out of this analysis because they only had one study.

When demographic and dosing regimen data were used for simulation, the Frymoyer model was identified as a generalizable model since it was able to match the reported concentrations (peak and trough) of other studies. Frymoyer et al. recommended an intravenous infusion of $4-5 \mathrm{mg} / \mathrm{kg}$ every $36 \mathrm{~h}$ to maintain gentamicin concentrations in the therapeutic range (peak around $10 \mathrm{mg} / \mathrm{dL}$, trough below $2 \mathrm{mg} / \mathrm{dL}$ ). The Frymoyer model is a onecompartment, first-order elimination model. BWT and serum creatinine $(\mathrm{SCr})$ were identified as influential covariates on $\mathrm{CL}{ }^{14}$ Based on the available studies, a BWT of $2.5-4 \mathrm{~kg}$ and an $\mathrm{SCr}$ of $0.3-1.5 \mathrm{mg} / \mathrm{dL}$ were chosen as a range for pharmacometric simulations. Peak and trough concentrations simulated using the Frymoyer (generalizable) model were within the range/mean SD of other studies. Comparison of simulated concentrations using the Frymoyer model against the observed/simulated concentrations of other studies is presented in Table 2. The recommended potential dosing regimen for individuals with BWT ranging from 2.5 to $4 \mathrm{~kg}$ and $\mathrm{SCr}$ values ranging from 0.2 to $1.5 \mathrm{mg} / \mathrm{dL}$ to maintain concentrations in the therapeutic range are presented in Table 3.

\section{DISCUSSION}

Clinical trials in neonates are the most challenging in situations like PA and TH (PATH), leading to a paucity of data on drug PK in such conditions. ${ }^{15}$ PopPK modeling is a better way to conduct research in vulnerable populations. ${ }^{16}$ Without going through the typical clinical trial process, PopPK studies successfully described the PK of medications and proposed dosing regimens. ${ }^{17}$ Many PopPK studies have recommended dosage regimen based on their study population data, but generalizable dosage regimen recommendation that works on wider populations are very sparse. The meta-modeling method was reported to help in the identification of a generalizable model that can be used to provide dosing recommendations in a variety of populations. ${ }^{18,19}$

Reduced $\mathrm{CL}$ during $\mathrm{TH}$ has been reported for amikacin, amoxicillin, and ampicillin. ${ }^{20-22}$ Seven reports on gentamicin during PATH were used in the meta-modeling procedure of the present study. The majority of the reports on gentamicin showed a reduction in $\mathrm{CL}$ during $\mathrm{TH}$, while there were reports of an increase and no change in $\mathrm{CL}$ as well. ${ }^{14,23-28} \mathrm{~A}$ recent metaanalysis reported that $\mathrm{CL}$ of gentamicin was reduced during $\mathrm{TH}^{29}$ In this study, they have summarized the results from the published studies and reported reduced $\mathrm{CL}$ of gentamicin during $\mathrm{TH}$. They focused on the combined impact of PA and TH. Since TH is PA's only therapeutic option, it is difficult to investigate the fundamental reason of reduced $\mathrm{CL}$. In the present study, we focused on the effects of PA and TH separately and the studies included in the present work ${ }^{26,27}$ had groups with and without TH during PA. In the present study, five reports ${ }^{14,23-25,28}$ compared the $\mathrm{CL}$ of neonates during PATH to the neonates without PA and TH. In 


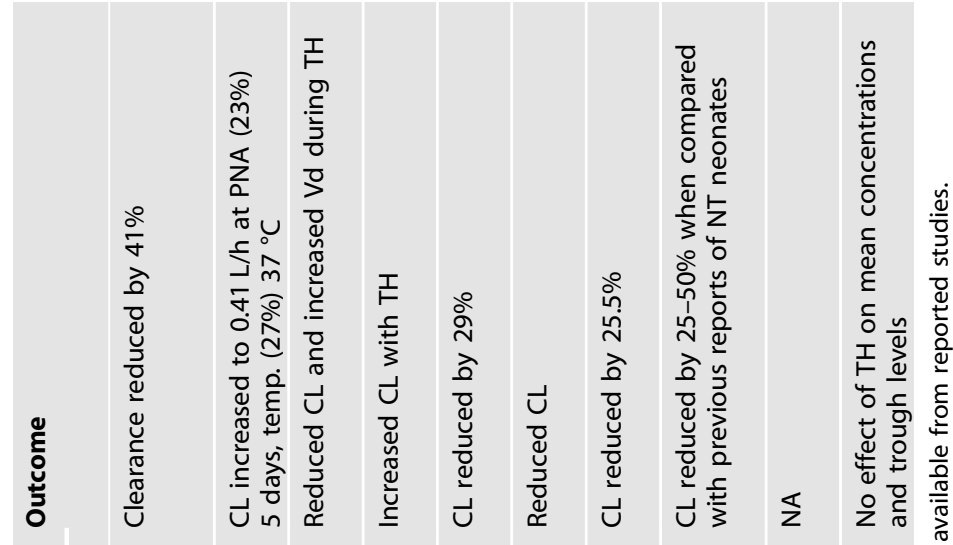

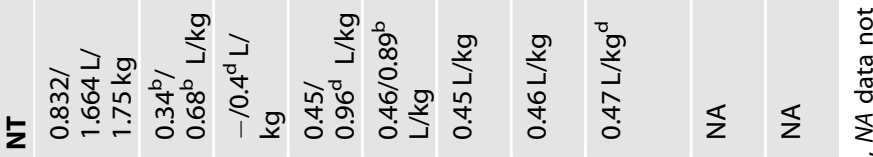

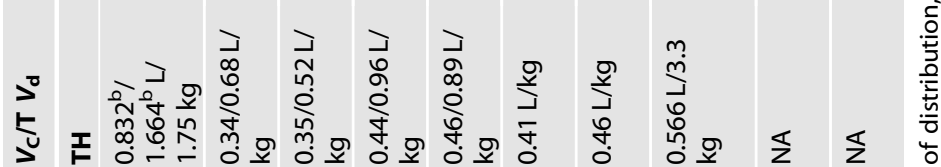

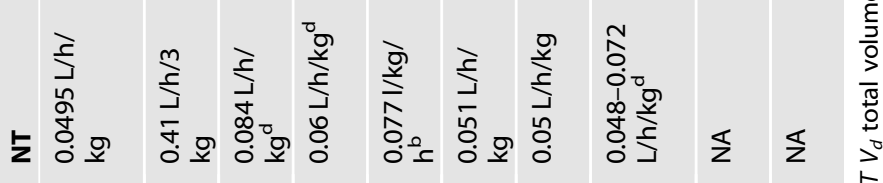

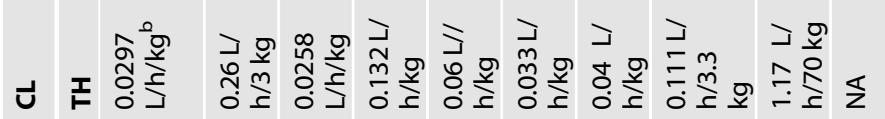

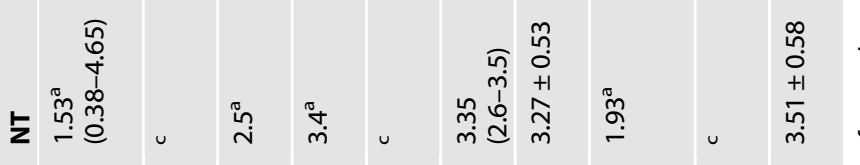

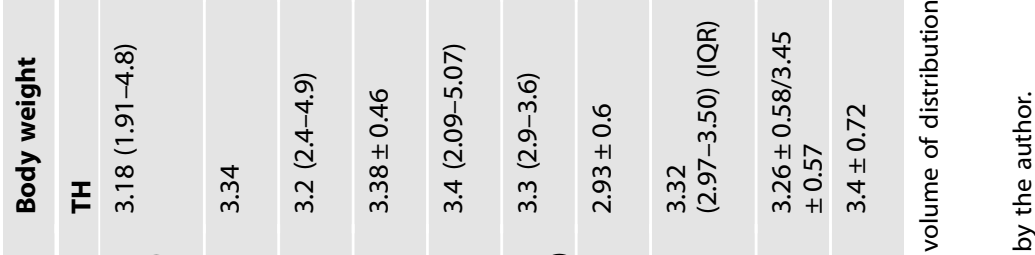

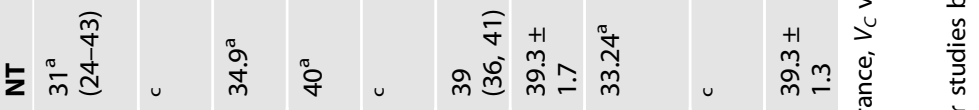

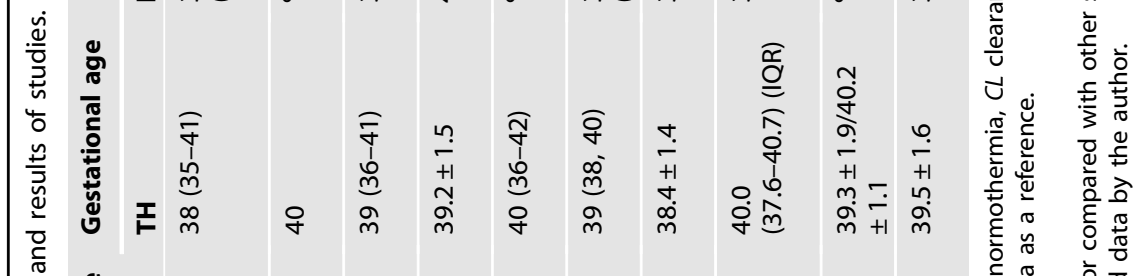

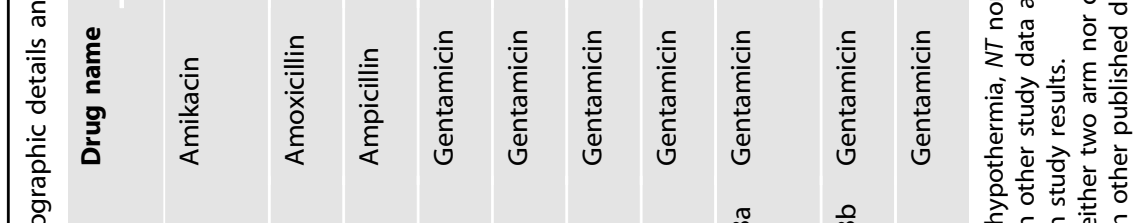

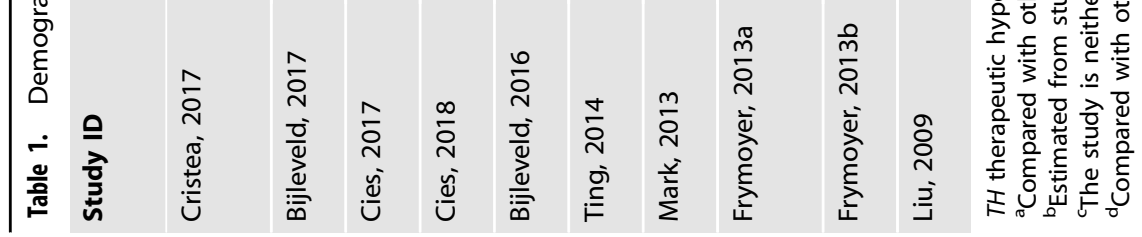


Table 2. Comparison of Frymoyer model simulated concentrations with other studies observed/simulated concentrations.

\begin{tabular}{|c|c|c|c|c|c|}
\hline \multirow[t]{2}{*}{ Study author } & \multirow[t]{2}{*}{ Mean/median doses as per studies } & \multicolumn{2}{|c|}{ Peak concentration (mg/L) } & \multicolumn{2}{|c|}{ Trough concentration (mg/L) } \\
\hline & & Reported & Simulated & Reported & Simulated \\
\hline Cies, 2018 & $5 \mathrm{mg} / \mathrm{kg}$ for $36 \mathrm{~h}$ & $10-12$ & 10.8 & $<2$ & 0.5 \\
\hline Ting, 2014 & $2.5 \mathrm{mg} / \mathrm{kg}$ for $12 \mathrm{~h}$ & $7.57-12.71$ & 8.3 & $2.29-5.52$ & 3.2 \\
\hline Mark, 2013 & $4 \mathrm{mg} / \mathrm{kg}$ for $24 \mathrm{~h}$ & $9.54 \pm 1.30$ & 9.54 & $1.68 \pm 0.69$ & 1.4 \\
\hline Frymoyer, 2013a & $5 \mathrm{mg} / \mathrm{kg} 36 \mathrm{~h}$ & $10.5(7.8-13.5)$ & 10.3 & $0.9(0.3-2)$ & 0.7 \\
\hline Liu 2009 & $4 \mathrm{mg} / \mathrm{kg}$ for $24 \mathrm{~h}$ & $>10$ & 10.7 & $2.19 \pm 1.7$ & 2.3 \\
\hline
\end{tabular}

Virtual subjects were generated for respective studies using their mean/median demographic details like birth weight, dose, dosing interval, and serum creatinine. The pharmacokinetic profile of these virtual subjects was simulated using Frymoyer model. ${ }^{14}$ Simulated concentrations were compared with the reported concentrations of respective studies.

Table 3. Dosage regimen recommendations to attain a therapeutic range of gentamicin.

\begin{tabular}{ll} 
SCr & $\begin{array}{l}\text { BWT (2.5-4 kg) } \\
\text { Gentamicin dosage of neonates based on per } \mathbf{k g} \text { of } \\
\text { birth weight }\end{array}$ \\
\hline $0.2-0.7 \mathrm{mg} / \mathrm{dL}$ & $4 \mathrm{mg} / \mathrm{kg} \mathrm{Q} 24 \mathrm{~h}$ \\
\hline $0.8-1.2 \mathrm{mg} / \mathrm{dL}$ & $4 \mathrm{mg} / \mathrm{kg} \mathrm{Q} 36 \mathrm{~h}$ \\
\hline $1.3-1.5 \mathrm{mg} / \mathrm{dL}$ & $4 \mathrm{mg} / \mathrm{kg} \mathrm{Q} 48 \mathrm{~h}$
\end{tabular}

Simulations for these recommendations were carried out for the serum creatinine from the range $0.2-1.5 \mathrm{mg} / \mathrm{dL}$ with the intervals of $0.1 \mathrm{mg} / \mathrm{dL}$. Birth weight ranges from 2.5 to $4 \mathrm{~kg}$ with intervals of $0.25 \mathrm{~kg}$. These recommendations are expected to result in a peak concentration between 7.5 and $10 \mu \mathrm{g} / \mathrm{mL}$ and trough concentrations between 0.5 and $2 \mu \mathrm{g} / \mathrm{mL}$.

these studies, the independent effect of PA or TH on CL could not be estimated as PA itself has been reported to cause renal impairment. $^{1,26,30,31}$

Two studies compared neonates diagnosed with PA against neonates with PATH with conflicting findings. ${ }^{26,27}$ Mark et al. reported that neonates with $\mathrm{PA}$ had higher $\mathrm{CL}$ than neonates undergoing PATH. ${ }^{27}$ When looked at the demography, neonates in PATH group had lower GA and BWT, although it was not statistically significant. GA and BWT are critical for renal maturation $^{32}$ and lower GA and BWT could have possibly contributed to reduced gentamicin CL. In another study, it was reported that there was no difference in $\mathrm{CL}$ between newborns with PA and PATH. ${ }^{26}$ There was no difference in terms of demographic characteristics like GA, BWT, and plasma creatinine in this study. According to the authors of this study, renal maturation is the key for $\mathrm{CL}$ of gentamicin, while $\mathrm{TH}$ has no effect on the $\mathrm{CL}$. ${ }^{26}$

$\mathrm{SCr}$ is commonly used to evaluate renal function, but in asphyxiated infants, $\mathrm{SCr}$ may not accurately reflect renal function. It is crucial to keep track of the rate at which $\mathrm{SCr}$ drops in order to find out how well those newborns' kidneys are functioning. ${ }^{33}$ As the rate of decline in $\mathrm{SCr}$ was not reported in any study, $\mathrm{SCr}$ measurement was the next best possibility to assess the renal function. To recommend gentamicin dose regimens to neonates with PA, it is important to consider GA, BWT, and SCr. Organ maturation can be explained by BWT, which is linearly connected to $\mathrm{GA} .^{34,35}$

One-compartment model with BWT and $\mathrm{SCr}$ as covariates on $\mathrm{CL}$ reported by Frymoyer et al. was successfully qualified by comparing with data from other studies. ${ }^{14}$ Adding $\mathrm{SCr}$ as a covariate in the model would help to understand the changes in renal function as gentamicin can induce renal toxicity even with shorter courses of high concentration dosage regimen. ${ }^{36,37}$ The Frymoyer et al. model was chosen as a generalizable model for two reasons: it successfully predicted concentrations reported in other papers and it has relevant covariates.

In two sets of population data, Sampson et al. validated the Frymoyer model's predictive performance. ${ }^{38}$ In one dataset, it performed well, and in another one, it did not do well. In the group where the Frymoyer model did not perform well, GA was significantly lower than the other group and it included premature neonates. Frymoyer study did not include premature infants in its model development. Therefore, failed validation has different explanations and these have to be considered when evaluating the Frymoyer model. The authors also mentioned that the model performance of the failed validation improved when post-therapy samples were dropped.

In their study, Frymoyer et al. ${ }^{14}$ simulated doses ranging from 3 to $5 \mathrm{mg} / \mathrm{kg}$ at intervals of $24-48 \mathrm{~h}$. Based on their simulation, the ideal dosing regimen was $4-5 \mathrm{mg} / \mathrm{kg}$ once every $36 \mathrm{~h}$. To arrive at the optimal dosage regimen, we used the range of BWT and $\mathrm{SCr}$ values reported across all studies, as well as dosage intervals, ranged from 24 to $48 \mathrm{~h}$. The current dosing recommendation is expected to reduce gentamicin trough exposure while maintaining optimal peak levels.

\section{Limitations}

The current recommendations need to be clinically validated in a prospective study. Differences in estimation methods of gentamicin and SCr may impact model prediction ability. Many studies used neonates without PA as controls to assess the effect of TH on $\mathrm{CL}$ and this is not a prudent approach. In some studies, premature neonates were included in the control arm and the inferences from these studies have to be assessed carefully. None of these studies were randomized controlled study.

\section{CONCLUSION}

For newborns undergoing PATH, available PopPK models of antimicrobial agents were collected. Gentamicin data were taken further for conducting a meta-model analysis. We identified the Frymoyer model as a generalizable model, which could match with data from other studies when simulations were carried out. BWT and SCr were found to be important covariates in this model. To maintain an appropriate therapeutic range, potential dosing regimens were recommended based on the value of covariates from the published studies. In infants receiving $\mathrm{TH}$, the gentamicin dosage was suggested from the range of $4 \mathrm{mg} / \mathrm{kg}$ every $24 \mathrm{~h}$ to 4 
$\mathrm{mg} / \mathrm{kg}$ every $48 \mathrm{~h}$. Prospective studies with these recommended regimens can aid in determining their effectiveness.

\section{REFERENCES}

1. Fattuoni, C., Palmas, F., Noto, A., Fanos, V. \& Barberini, L. Perinatal asphyxia: a review from a metabolomics perspective. Molecules 20, 7000-7016 (2015).

2. Ahearne, C. E., Boylan, G. B. \& Murray, D. M. Short and long term prognosis in perinatal asphyxia: an update. World J. Clin. Pediatr. 5, 67-74 (2016).

3. Giannoni, E. et al. Neonatal Sepsis of early onset, and hospital-acquired and community-acquired late onset: a Prospective Population-Based Cohort Study. J. Pediatr. 201, 106-114 (2018).

4. Chiang, M. C., Jong, Y. J. \& Lin, C. H. Therapeutic hypothermia for neonates with hypoxic ischemic encephalopathy. Pediatr. Neonatol. 58, 475-483 (2017).

5. Datta, V. Therapeutic hypothermia for birth asphyxia in neonates. Indian J. Pediatr. 84, 219-226 (2017).

6. Lemyre, B. \& Chau, V. Hypothermia for newborns with hypoxic-ischemic encephalopathy. Paediatr. Child Health 23, 285-291 (2018).

7. Silveira, R. C. \& Procianoy, R. S. Hypothermia therapy for newborns with hypoxic ischemic encephalopathy. J. Pediatr. 91, S78-S83 (2015).

8. Zanelli, S., Buck, M. \& Fairchild, K. Physiologic and pharmacologic considerations for hypothermia therapy in neonates. J. Perinatol. 31, 377-386 (2011).

9. Tanigasalam, V., Bhat, V., Adhisivam, B. \& Sridhar, M. G. Does therapeutic hypothermia reduce acute kidney injury among term neonates with perinatal asphyxia? - a randomized controlled trial. J. Matern. Fetal Neonatal Med. 29, 2545-2548 (2016).

10. Solevag, A. L., Schmolzer, G. M. \& Cheung, P. Y. Novel interventions to reduce oxidative-stress related brain injury in neonatal asphyxia. Free Radic. Biol. Med. 142, 113-122 (2019).

11. Sheiner, L. B. The population approach to pharmacokinetic data analysis: rationale and standard data analysis methods. Drug Metab. Rev. 15, 153-171 (1984).

12. Bonate, P. L. Clinical trial simulation in drug development. Pharm. Res. 17, 252-256 (2000).

13. Rackauckas, C. et al. Accelerated predictive healthcare analytics with pumas, a high performance pharmaceutical modeling and simulation platform. bioRxiv https://doi.org/10.1101/2020.11.28.402297 (2020).

14. Frymoyer, A., Meng, L., Bonifacio, S. L., Verotta, D. \& Guglielmo, B. J. Gentamicin pharmacokinetics and dosing in neonates with hypoxic ischemic encephalopathy receiving hypothermia. Pharmacotherapy 33, 718-726 (2013).

15. Coppini, R., Simons, S. H. P., Mugelli, A. \& Allegaert, K. Clinical research in neonates and infants: challenges and perspectives. Pharm. Res. 108, 80-87 (2016).

16. Patel, P. et al. Dried blood spots and sparse sampling: a practical approach to estimating pharmacokinetic parameters of caffeine in preterm infants. Br. J. Clin. Pharmacol. 75, 805-813 (2013).

17. Dahmane, E. et al. Clinical pharmacology-driven translational research to optimize bedside therapeutics of sotalol therapy. Clin. Transl. Sci. 12, 648-656 (2019).

18. Liu, T., Ivaturi, V. \& Gobburu, J. Integrated model to describe morphine pharmacokinetics in humans. J. Clin. Pharmacol. 59, 1070-1077 (2019).

19. Liu, T., Lewis, T., Gauda, E., Gobburu, J. \& Ivaturi, V. Mechanistic population pharmacokinetics of morphine in neonates with abstinence syndrome after oral administration of diluted tincture of opium. J. Clin. Pharmacol. 56, 1009-1018 (2016).

20. Bijleveld, Y. A. et al. Population pharmacokinetics of amoxicillin in term neonates undergoing moderate hypothermia. Clin. Pharm. Ther. 103, 458-467 (2018).

21. Cies, J. J., Fugarolas, K. N., Moore, W. S., Mason, R. W. \& Menkiti, O. R. Population pharmacokinetics and pharmacodynamic target attainment of ampicillin in neonates with hypoxemic-ischemic encephalopathy in the setting of controlled hypothermia. Pharmacotherapy 37, 456-463 (2017).

22. Cristea, S. et al. Amikacin pharmacokinetics to optimize dosing in neonates with perinatal asphyxia treated with hypothermia. Antimicrob. Agents Chemother. 61, e01282-17 (2017).

23. Bijleveld, Y. A. et al. Altered gentamicin pharmacokinetics in term neonates undergoing controlled hypothermia. Br. J. Clin. Pharmacol. 81, 1067-1077 (2016).

24. Cies, J. J., Habib, T., Bains, V., Young, M. \& Menkiti, O. R. Population pharmacokinetics of gentamicin in neonates with hypoxemic-ischemic encephalopathy receiving controlled hypothermia. Pharmacotherapy 38, 1120-1129 (2018).

25. Frymoyer, A. et al. Every $36-\mathrm{h}$ gentamicin dosing in neonates with hypoxicischemic encephalopathy receiving hypothermia. J. Perinatol. 33, 778-782 (2013).

26. Liu, X., Borooah, M., Stone, J., Chakkarapani, E. \& Thoresen, M. Serum gentamicin concentrations in encephalopathic infants are not affected by therapeutic hypothermia. Pediatrics 124, 310-315 (2009).

27. Mark, L. F., Solomon, A., Northington, F. J. \& Lee, C. K. Gentamicin pharmacokinetics in neonates undergoing therapeutic hypothermia. Ther. Drug Monit. 35, 217-222 (2013).
28. Ting, J. Y., Kwan, E., McDougal, A. \& Osiovich, H. Pharmacokinetics of gentamicin in newborns with moderate-to-severe hypoxic-ischemic encephalopathy undergoing therapeutic hypothermia. Indian J. Pediatr. 82, 119-125 (2015).

29. Choi, D. W., Park, J. H., Lee, S. Y. \& An, S. H. Effect of hypothermia treatment on gentamicin pharmacokinetics in neonates with hypoxic-ischaemic encephalopathy: a systematic review and meta-analysis. J. Clin. Pharm. Ther. 43, 484-492 (2018).

30. Alaro, D., Bashir, A., Musoke, R. \& Wanaiana, L. Prevalence and outcomes of acute kidney injury in term neonates with perinatal asphyxia. Afr. Health Sci. 14 682-688 (2014).

31. Nouri, S. et al. Acute renal failure in full term neonates with perinatal asphyxia. Prospective study of 87 cases. Arch. Pediatr. 15, 229-235 (2008).

32. Rhodin, M. M. et al. Human renal function maturation: a quantitative description using weight and postmenstrual age. Pediatr. Nephrol. 24, 67-76 (2009).

33. Gupta, C., Massaro, A. N. \& Ray, P. E. A new approach to define acute kidney injury in term newborns with hypoxic ischemic encephalopathy. Pediatr. Nephrol. 31, 1167-1178 (2016).

34. De Cock, R. F. et al. Maturation of the glomerular filtration rate in neonates, as reflected by amikacin clearance. Clin. Pharmacokinet. 51, 105-117 (2012).

35. Topcu, H. O. et al. Birth weight for gestational age: a reference study in a tertiary referral hospital in the middle region of Turkey. J. Chin. Med. Assoc. 77, 578-582 (2014).

36. McWilliam, S. J., Antoine, D. J., Smyth, R. L. \& Pirmohamed, M. Aminoglycosideinduced nephrotoxicity in children. Pediatr. Nephrol. 32, 2015-2025 (2017).

37. Siber, G. R., Echeverria, P., Smith, A. L., Paisley, J. W. \& Smith, D. H. Pharmacokinetics of gentamicin in children and adults. J. Infect. Dis. 132, 637-651 (1975).

38. Sampson, M. R. et al. Predictive performance of a gentamicin population pharmacokinetic model in neonates receiving full-body hypothermia. Ther. Drug Monit. 36, 584-589 (2014).

\section{ACKNOWLEDGEMENTS}

We gratefully acknowledge the help of a BMS Foundation Ph.D. fellowship and Dr. Vijay Ivaturi for introducing us to the concept of generalizable models for dosage regimen design.

\section{AUTHOR CONTRIBUTIONS}

All the authors significantly contributed to each phase of study conduct and manuscript writing. S.M. and E.A.R. took the lead in the systematic search and screening of the articles. R.M., A.P., and S.M. led the process of identifying a generalizable model using pharmacometric simulations. R.V. and S.R.M. led the process of analyzing and interpreting the data. S.M. and A.P. led the pharmacometric simulations to identify the dosage regimens under the guidance of S.R.M. and L.E.L. L.E.L., R.V., and S.R.M. gave critical and conceptual inputs for developing the optimized dosage regimens. All the authors have repeated the whole work to check the reproducibility of the work. R.V., L.E.L., and S.R.M. have given the final approval of the version to be published. S.R.M. has supervised the whole work throughout the project.

\section{FUNDING INFORMATION}

The first author (S.M.) has received BMS Foundation Ph.D. fellowship. Open access funding provided by Manipal Academy of Higher Education, Manipal.

\section{COMPETING INTERESTS}

The authors declare no competing interests.

\section{ADDITIONAL INFORMATION}

Supplementary information The online version contains supplementary material available at https://doi.org/10.1038/s41390-021-01714-0.

Correspondence and requests for materials should be addressed to Surulivelrajan Mallayasamy.

Reprints and permission information is available at http://www.nature.com/ reprints

Publisher's note Springer Nature remains neutral with regard to jurisdictional claims in published maps and institutional affiliations. 
(c) Open Access This article is licensed under a Creative Commons

Attribution 4.0 International License, which permits use, sharing, adaptation, distribution and reproduction in any medium or format, as long as you give appropriate credit to the original author(s) and the source, provide a link to the Creative Commons license, and indicate if changes were made. The images or other third party material in this article are included in the article's Creative Commons license, unless indicated otherwise in a credit line to the material. If material is not included in the article's Creative Commons license and your intended use is not permitted by statutory regulation or exceeds the permitted use, you will need to obtain permission directly from the copyright holder. To view a copy of this license, visit http://creativecommons. org/licenses/by/4.0/.

(c) The Author(s) 2021 